Contaminats

\title{
Light microscopy with a differential staining technique for the characterization and discrimination of insects versus marine arthropods in processed animal proteins
}

\author{
Matteo Ottoboni, Marco Tretola, Federica Cheli, Daniela Marchis, Pascal \\ Veys, Vincent Baeten \& Luciano Pinotti
}

To cite this article: Matteo Ottoboni, Marco Tretola, Federica Cheli, Daniela Marchis, Pascal Veys, Vincent Baeten \& Luciano Pinotti (2017): Light microscopy with a differential staining technique for the characterization and discrimination of insects versus marine arthropods in processed animal proteins, Food Additives \& Contaminants: Part A, DOI: 10.1080/19440049.2016.1278464

To link to this article: http://dx.doi.org/10.1080/19440049.2016.1278464

Accepted author version posted online: 20 Jan 2017.

Submit your article to this journal ๘

山 Article views: 1

View related articles

View Crossmark data $₫$ 
Publisher: Taylor \& Francis \& Informa UK Limited, trading as Taylor \& Francis

Journal: Food Additives \& Contaminants: Part A

DOI: $10.1080 / 19440049.2016 .1278464$

Light microscopy with a differential staining technique for the characterization and discrimination of insects versus marine arthropods in processed animal proteins

Matteo Ottoboni1, Marco Tretola1, Federica Cheli1, Daniela Marchis2, Pascal Veys3, Vincent Baeten3, Luciano Pinotti1

1 Department of Health, Animal Science and Food Safety VESPA, Università degli Studi di Milano, Milan, Italy.

2 National Reference Laboratory on animal proteins in feed, Istituto Zooprofilattico Sperimentale di Piemonte, Liguria e Valle D'Aosta, Italy.

3 Food and Feed Quality Unit, Valorisation Department, Walloon Agricultural Research Centre CRA-W, Gembloux, Belgium.

Corresponding author: Prof. Luciano Pinotti e-mail: luciano.pinotti@unimi.it

The aim of this study was to evaluate the use of light microscopy with a differential staining technique for the discrimination of insect material from marine arthropods - classified as fishmeal. Specifically, three samples of single species insect material, Hermetia illucens (HI), Bombyx mori (BM) and Tenebrio molitor (TM) and two samples of marine arthropods, shrimp material and krill, have been analysed and compared after staining by two reagents to enhance fragment identification. Alizarin Red (AR) and Chlorazol black (CB), which react respectively with calcium salts and chitin, were tested for their potential efficacy in distinguishing between insect and marine materials. Results indicated that AR failed to stain HI, BM and TM materials. By contrast, the three insect species materials tested (HI, BM and TM) were stained by CB. When shrimp fragments and krill were considered, AR and CB stained marine materials reddish-pink and light blue to black, respectively. By combining these results it can be suggested 
that CB staining may efficiently be used to mark insect materials; AR does stain shrimp fragments but does not stain the tested insect material, indicating a possible approach for discriminating between insects and marine arthropods. However, since the present study has only been done on pure materials and on a small set of samples, possible implementation of this technique still needs to be confirmed in complex matrices such as compound feed.

Keywords: insect meals; arthropods; microscopy; staining; identification.

\section{Introduction}

In several Member States within the European Union, numerous studies are running on insect rearing and biomass production for feed purpose. Ongoing researches on insects as feed are mainly focused on types of feed substrates to raise the insects, nutritional values of the produced insects, diet formulations, and animal performance when these materials are fed (Makkar, 2014; Barroso, 2014; van Huis 2013; Veldkamp et al. 2015; Sánchez-Muros et al., 2014; Rumpold and Schlüter, 2013). The potential use of insect meal as a feed ingredient for farmed animals has been proposed mainly as a protein source (Veldkamp et al., 2015).

However, the major barrier to growth of the edible insect sector is the absence of precise and insect-focused legislation, as reported elsewhere (FAO, 2013; Lahteenmaki Uutela \& Grmelova, 2016).

As reported by the recent EFSA (2015) document "Risk profile of insects as food and feed", there are several legislative requirements that impact the use of insects as food and feed (at EU level). Currently, the feed ban provisions of Regulation (EC) No 999/2001 (TSE Regulation) do not allow insect meals to be fed to farmed animals due to lack of a safety profile. Furthermore, with respect to feed/substrate for insects, Annex III to Regulation (EC) No 767/2009 prohibits the feeding of faeces and separated digestive tract content even though these materials are used in other parts of the world as substrates in insect production. These aspects are relevant for insect material because insect meals should be considered as processed animal proteins (PAPs). The legislative framework specifically related to insects used as food and feed, however, is still under development. Beyond categorization and destination concerns about insect meals, a 
further step in defining future insect legislation is the implementation of analytical methods, for their traceability and identification.

Nowadays, insect materials in feed and food matrices have been considered as contaminants and/or extraneous matter. The standard method for determining insect fragments in flours for human consumption (AOAC method 993.26), is based on insect fragments extraction by acid digestion and flotation, but this technique is laborious and time consuming. There are a few methods that have sought to determine insect fragment counts using enzyme-linked immunosorbent assays (ELISA) (Quinn et al., 1992; Schatzki et al., 1993; Brader et al., 2002), DNA finger printing (Balasubramanian et al., 2007) or near-infrared spectroscopy (NIRS) (Perez-Mendoza et al., 2003). A further approach has been proposed by Bhuvaneswari and co-workers (2011). In the latter study, authors have compared the speck counts from an electronic speck counter, acid hydrolysis and flotation (AOAC method 993.26), and near-infrared (NIR) hyperspectral imaging in experimentally contaminated semolina. However, in this case too the detection of insect as extraneous matters was the goal.

Assuming that insect meals will be considered as PAPs, and therefore as a feed ingredient, the already existing methods for PAP detection and identification can be considered as a robust starting point. In the EU, although several techniques have been proposed (van Raamsdonk et al., 2012; Veys et al., 2012; Fumière et al., 2009; Ottoboni et al., 2014; Pinotti et al., 2013; Tena et al., 2014; Pinotti et al., 2016), only two methods are allowed within the framework of official controls for the detection of animal proteins in feed, namely light microscopy and polymerase chain reaction (PCR) (Regulation EC No 152/2009; Regulation EU No 51/2013). Both methods have been validated for proper implementation of the feed ban.

In the case of light microscopy, Alizarin Red (AR) has been authorized as a staining reagent in Reg. EU 51/2013 (European Commission, 2013b) for the official control of feed. The staining reagent colours some major mineral constituents, like hydroxyapatite and calcium phosphates (e.g., tricalcium phosphate), both well represented in bones (EURL-AP, 2013; Liu et al., 2011). Assuming an adaptation of the microscopic method for other PAPs, such as insect material, several aspects must be considered. First, the nature of the insect materials, which is variable (Makkar et al., 2014). Insect particles present different features according to the physiological stage of the insects (e.g. larvae 
or adult state) used for producing the meals (Finke, 2009). Second, absence of bones as well as presence of an exoskeleton (e.g. cuticle structure), make these materials in some circumstance quite close to marine arthropods (e.g. shrimps). In term of composition however, shrimp and krill exoskeleton is naturally rich in calcium (Watkins et al., 1982; Chen et al., 2009), while a small amount of calcium is present in Hermetia illucens larvae (Finke et al., 2013). This implies that authorized staining reagents, like AR, would not be suitable for insect materials.

In this context, chlorazol black (CB) is a stain with a high affinity for chitin, a unique structural homopolymer polysaccharide of $\beta$-[1,4]-linked D-N-acetylglucosamine (Thomas et al., 2008). As reported by Finke (2009) Hermetia illucens is rich in chitin, while for the other insect material its presence can be variable (Makkar 2014). Chitin in combination with calcium deposits is also one of the main components in shrimp and krill exoskeletons (Watkins et al., 1982; Al Sagheer et al., 2009; Chen et al., 2009 Nicol and Hosie, 1993). This makes CB a potential staining reagent for both insect and marine arthropod identification.

Starting from these assumptions, the aim of this study was to evaluate the use of light microscopy and the potential of two different staining reagents, namely $\mathrm{AR}$ and $\mathrm{CB}$, for the discrimination of insect PAP against marine arthropods - classified as fishmeal.

\section{Material and method}

Nine different animal meals samples were selected. Specifically, 7 samples of insect material and 2 samples of marine arthropods were used. Insect samples were obtained from the following species: black soldier fly larvae (Hermetia illucens, 4 samples provided by European Union Reference Laboratory for Animal Proteins in feedingstuffs, EURL-AP), silkworm (Bombyx mori, 1 sample provided by Centro di Referenza Nazionale per la Sorveglianza e il Controllo degli Alimenti per gli Animali, C.Re.A.A.), and mealworm (Tenebrio molitor, 1 sample provided by EURL-AP and 1 sample provided by C.Re.A.A.). All insect samples were pure materials obtained under experimental and lab scale conditions. In the case of marine arthropods a shrimp meal and a krill meal sample were used, both provided by EURL-AP. All dried pure samples were ground with a mortar and pestle. Subsequently, for each sample at least 3 microscopic slides were prepared using Norland Optical adhesive 65 or glycerol as the 
embedding agent as indicated in the official method for the determination of constituents of animal origin in feed. After curing, slides were examined using a compound microscope (Olympus BX41, Tokyo, Japan or Carl Zeiss Axio Imager A1) at several magnifications under bright field conditions. Both insect and marine fragment images were acquired using a digital camera (CoolSNAP-Pro cf Color or AxioCam MRc coupled with a 0.63 port).

In a second step and in order to enhance fragment identification, all samples were analyzed using $\mathrm{AR}$ and $\mathrm{CB}$ as staining reagents. Briefly AR staining (Color Index Number 58005, Sigma-Aldrich 3050 Spruce Street, Saint Louis, MO 63103, USA) was performed according to Annex VI of EC/152/2009. The stained material was then placed in an oven at $68^{\circ} \mathrm{C}$ until completely dry. Subsequently, for each sample at least 3 microscopic slides were prepared using Norland Optical adhesive 65 or glycerol as the embedding agent. In the case of CB stain (Color Index Number 30235, Sigma-Aldrich 3050 Spruce Street, Saint Louis, MO 63103, USA), dried samples (100 mg) were transferred into a glass test tube and rinsed twice with approximately $5 \mathrm{ml}$ ethanol (each time a vortex was used; the solvent was allowed to settle approximately one minute and then poured off). Before using this staining reagent, the sample was bleached by adding at least $1 \mathrm{ml}$ sodium hypochlorite solution. The reaction was allowed to continue for 10 minutes. Next, the tube was filled with water, the sample was left to settle for 2-3 minutes, and the water and any suspended particles were poured off. The sample was rinsed twice more with approximately $10 \mathrm{ml}$ of water (each time a vortex was used; the mixture was allowed to settle approximately one minute and the supernatant poured off). The sample was rinsed once with approximately $5 \mathrm{ml}$ acetone, vortexed and decanted, left to settle, and then the acetone was poured off. A few drops (depending on the amount of residue) of the CB solution were added. The mixture was shaken and left a few seconds for the staining to occur reaction. The coloured sediment was rinsed twice with approximately $5 \mathrm{ml}$ ethanol followed by three rinses with acetone (each time a vortex was used; the supernatant was allowed to settle approximately one minute and then poured off). The sample was placed in an oven at $68^{\circ} \mathrm{C}$ until completely dry.

\section{Results and discussion}

Results obtained in the present study are reported in Figure 1 (from A to I) for insect materials and in Figure 2 (from A to F) for marine arthropods materials. For each 
sample a selected picture without (microscopic slides mounted with Norland Optical adhesive 65 or glycerol) or with specific staining reagents have been presented. In general, when any staining reagent had been used, distinguishing between insect and marine arthropods materials was difficult. No substantial differences were observed between Hermetia illucens, Tenebrio Molitor and Bombyx mori vs krill and shrimps samples. As shown in Figures 1A to 1C Hermetia illucens fragments of the exoskeleton are characterized by cell-like structures (Fig. 1A, 1B and 1C) four- or five-sided with thick walls and a broad lumen. This lumen, as observed in surface view, is wider than the thick walls (see arrowhead in Figure 1C). The structure of the thick walls gives the cells a honeycomb-like appearance. Hermetia illucens material colours ranges from grey-cream to brown and dark. Bristles, generally long, narrow and yellow-brownish, have also been observed in Hermetia illucens material (Fig. 1A, 1B and 1C). Notably, bristles can present in different colours, from the proximal part to the distal one or from the inner part to the peripheral one. Colour moves through yellow shades, followed by a black and yellow line in the middle. In the same preparation other pyramidal structures have been observed, but their precise characterization is difficult and speculative at this stage: further investigation are needed in order to define their specific features.

In Bombyx mori cuticular fragments (Fig. 1D, 1E and 1F) a similar pattern to that reported for $\mathrm{HI}$ has been observed, color moves from yellow (background) to brown (broad lumen). Differently to HI no bristles were observed in Bombyx mori samples.

In the case of Tenebrio molitor (Fig. 1G, $1 \mathrm{H}$ and 1I) material no specific pattern nor bristles were observed. However in all TM fragments rare dark pigmented brownish dots were observed. These structures are similar to glandular pores in ventral abdominal cuticle of Tenebrio molitor larvae described by Locke (1961). Tenebrio molitor material varied from grey to deep amber-brown in color.

Marine arthropod material (shrimp and krill) is presented in Figures 2A to 2F. Under the microscope, it can be observed that when any staining reagent had been used (microscopic slides mounted with Norland Optical adhesive 65 or glycerol) fragment transparency could be seen. Most of the features observed not only are in line with the literature (Makowski et al., 2011), but in some cases quite similar to those observed in insect samples. Krill and shrimps fragments were characterized by the presence of more-or-less transparent particles of the chitinous shells. These particles showed very 
fine lines intersecting at random angles and extending across the whole particle.

Occasionally, lines would connect across several other lines, forming triangles and other geometric shapes (Fig. 2D and 2E). In some areas, there may be cross-hatching (Fig.

$2 \mathrm{~A}, 2 \mathrm{D}, 2 \mathrm{E}$ and $2 \mathrm{~F})$. These findings are in line with the description of shrimp and krill meals reported by Makowski et al. (2011).

Results obtained with AR staining of insect fragments are also reported in Figure 1. It can be observed that no staining reaction was observed for insect fragments. AR stains calcium ions in several mineral forms. Specifically, it has been reported that this staining reacts principally with hydroxyapatite (contained in bone) but also with calcium phosphates (e.g., tricalcium phosphate) (EURL-AP, 2013). Accordingly, the hypothesis behind this experiment was that these insect species could be coloured using a calcium specific stain. Indeed, calcium content in Hermetia illucens larvae can be higher than $75 \mathrm{~g} / \mathrm{kg}$ DM (Makkar et al., 2014). Nevertheless, after staining Hermetia illucens material with the alizarin solution, no coloration was observed (Fig. 1A). This phenomenon can be ascribed to different factors: i) there were no calcium salts present in HI larvae sample; ii) the mineral form of calcium contained in this insect species does not react with AR; iii) the calcium salts were not accessible to the dye, because for instance of the presence of lipid-like waxes, iv) the fact that in the present study insect material of each species was collected at larval stages that do not have a fully differentiated cuticle possibly not containing calcium ions. However, a combination of all factors cannot be excluded. The same absence of staining was found for silkworm (Bombyx mori, 1 sample), and mealworm (Tenebrio molitor, 1 sample) (Fig. 1D and 1G respectively), even though some differences in calcium content, compared to HI, have been reported in literature for these species (Makkar et al., 2014).

With regard to the $\mathrm{CB}$ stain test performed on $\mathrm{HI}$, results obtained evidenced that the insect materials stained dark black (Fig. 1B). Chlorazol black is a dye with a high affinity for chitin (Thomas et al., 2008), which is abundant in Hermetia illucens (Finke, 2009). With silkworms and mealworms the same reaction has been observed: insect material became blue/black (Fig. 1E and 1H).

Moving to marine arthropods, it can be observed that AR and CB stain tests, coloured the shrimp fragments reddish-pink (Fig. 2A) and dark black (Fig. 2B), respectively. These results were expected because the shrimp exoskeleton is naturally rich in both 
chitin and calcium (Watkins et al., 1982; Al Sagheer et al., 2009). In the case of krill, fragments have shown a reddish-pink staining for AR staining and a light blue/black for CB staining. When using AR, results obtained for krill were comparable to shrimp (Fig. 2D), but different for Chlorazol black. The CB staining has shown a limited reaction in the krill sample (see Fig. 2E). This latter aspect merits further investigations, because the assumption is that both marine materials are characterized by similar compositions

By combining these results (Table 1), it can be suggested that $\mathrm{CB}$ stain is not adequate to distinguish between terrestrial (insect) and marine (shrimp and krill) arthropods. both materials get stained with CB. This represents a limit to the potential of these staining agents for insect material identification in complex matrices such as compound feed. Alizarin Red does stain shrimp fragments but did not stain the tested insect material, indicating a possible approach for discriminating between terrestrial and marine arthropods. However, further progress in this area requires the establishment of a sufficiently large and representative reference materials bank, which should also contain heat treated and processed insect meals. In fact, one of the weakness of this study was not only the limited number of samples tested, but also the mild heat treatment (drying only) that they have received. This was principally due to the difficulty in obtaining pure insect samples. In spite of that, the results presented here may represent a good starting point for future research in the field of feed safety.

\section{Conclusion}

This study has investigated the use of light microscopy and two selected stains in order to enhance features that could allow distinguishing insect material from marine arthropods materials. Results obtained in the present study indicated that microscopy has some potential for this when specific staining reagents are used. By contrast, when any staining reagent was used (microscopic slides mounted with Norland Optical adhesive 65 or glycerol), distinguishing between insect and marine arthropods materials was difficult. No substantial differences were observed between Hermetia illucens, Tenebrio Molitor and Bombyx mori vs krill and shrimps samples. The use of Alizarin red can help in the recognition of insect fragments in comparison with marine arthropods, although with some limits. Composition of insect meals, which is strongly correlated to physiological state (i.e. larval vs adult form) and growth condition (i.e. substrate) of the insect, may affect staining reactions and in turn identification of the 
material. Thus, although the tools proposed here appear promising, a combined approach which include molecular methods (PCR) or others (e.g. NIRM), is therefore recommended.

\section{References}

Al Sagheer FA, Al-Sughayer MA, Muslim S, Elsabee MZ. 2009. Extraction and characterization of chitin and chitosan from marine sources in Arabian Gulf. Carbohydrate Polymers 77, 410-419.

AOAC 993.26. Association Of Analytical Communities. 1996. Test Method: Light Filth in Whole Wheat Flour - Flotation M.

Barroso FG, de Haro C, Sánchez-Muros MJ, Venegas E, Sánchez AM, Bañón CP. 2014. The potential of various insect species for use as food for fish. Aquaculture 422-423, 193-201.

Balasubramanian A, Jayas DS, Fernando WGD, Li G, White NDG. 2007. Sensitivity analysis of DNA fingerprinting technique for detecting insect fragments in wheat flour. Canadian Biosystems Engineering 49, 41-45.

Brader B, Lee RC, Plarre R, Burkholder WG, Kitto BG, Kao C, Polston L, Dorneanu E, Szabo I, Mead B, Rouse B, Sullins D, Denning R. 2002. A comparison of screening methods for insect contamination in wheat. Journal of Stored Products Research 38, 75-86.

Bhuvaneswari K, Fields PG, White NDG, Sarkar AK, Singh CB, Jayas DS. 2011. Image analysis for detecting insect fragments in semolina. Journal of Stored Products Research 47, 20-24.

Chen YC, Tou JC, Jaczynski J. 2009. Amino acid and mineral composition of protein and other components and their recovery yields from whole Antarctic krill (Euphausia superba) using isoelectric solubilization/precipitation. JOURNAL OF FOOD SCIENCE 74 (2), 31-39.

EFSA, 2015. European Food Safety Authority. Risk profile related to production and consumption of insects as food and feed. EFSA Journal 13 (10), 4257.

EURL-AP, 2013. European Union Reference Laboratory for Animal Proteins in feedingstuffs, EURL-AP Standard Operating Procedure, Use of staining reagents. Publication date 28.03.2013. Available at: http://eurl.craw.eu/img/page/sops/EURLAP\%20SOP\%20use\%20of\%20staining\%20reagents\%20V1.0.pdf. 
European Commission, 2001. Regulation No. 999/2001 of 22 May 2001 laying down rules for the prevention, control and eradication of certain transmissible spongiform encephalopathies. In: Official Journal, L 147, 31/05/2001, pp 1-40. European Commission, 2009a. Regulation No. 152/2009 of 27 January 2009 laying down the methods of sampling and analysis for the official control of feed. In: Official Journal, L54, 26/02/2009 2009. pp. 1-130.

European Commission, 2009b. Regulation No. 767/2009 of 13 July 2009 on the placing on the market and use of feed, amending European Parliament and Council Regulation (EC) No 1831/2003 and repealing Council Directive 79/373/EEC, Commission Directive 80/511/EEC, Council Directives 82/471/EEC, 83/228/EEC, 93/74/EEC, 93/113/EC and 96/25/EC and Commission Decision 2004/217/EC. In Official Journal, L 229, 1.9.2009, pp 1-28.

European Commission, 2013a. Regulation No. 56/2013 of 16 January 2013 amending Annexes I and IV to Regulation No 999/2001 laying down rules for the prevention, control and eradication of certain transmissible spongiform encephalopathies. In: Official Journal, L21, 24/01/2013. pp. 3-16.

European Commission, 2013b. Regulation No. 51/2013 of 16 January 2013 amending Regulation No 152/2009 as regards the methods of analysis for the determination of constituents of animal origin for the official control of feed. In: Official Journal, L 20, 23/01/2013. pp. 33-43.

FAO, 2013. Food and Agriculture Organization of the United Nations. THE STATE OF FOOD AND AGRICULTURE 2013, Rome. Available at: http://www.fao.org/docrep/018/i3300e/i3300e00.htm

Finke MD. 2009. Estimate of Chitin in Raw Whole Insects. Zoo Biology 26, 105-115. Finke MD. 2013. Complete Nutrient Content of Four Species of Feeder Insects. Zoo Biology 32, 27-36.

Fumière O, Veys P, Boix A, Baeten V, Berben G. 2009. Methods of detection, species identification and quantification of processed animal proteins in feedingstuffs. Biotechnologie, Agronomie, Société et Environnement 13 (s), 59-70.

Lahteenmaki-Uutela A, Grmelova N. 2016. European Law on Insects in Food and Feed. European Food \& Feed Law Review, 11 (1), 2-8.

Liu X, Han L, Veys P, Baeten V, Jiang X, Dardenne P. 2011. An overview of the legislation and light microscopy for detection of Processed Animal Proteins in feeds. Microscopy Research and Technique 74(8), 735-743. 
Locke M. 1961. Pore canals and related structures in insect cuticle. The Journal of biophysical and biochemical cytology, 10(4), 589-618.

Makkar HPS, Tran G, Heuzé V, Ankers P. 2014. State-of-the-art on use of insects as animal feed. Animal Feed Science and Technology 197, 1-33.

Makowski J, Vary N, McCurtcheon M, Veys P, 2011. Microscopic analysis of agricultural products, 4th edition. Urbana, USA. AOCS Press.

Nicol S, Hosie GW. 1993. Chitin production by krill. Biochemical systematics and Ecology, 21 (2), 181-184.

Ottoboni M, Cheli F, Amato G, Marchis D, Brusa B, Abete MC, Pinotti L, 2014. Microscopy and image analysis based approaches for the species-specific identification of bovine and swine bone containing material Ital J Anim Sci 13(3187), 377-381.

Perez-Mendoza P, Throne JE, Dowell FE, Baker JE. 2003. Detection of insect fragments in wheat flour by near-infrared spectroscopy. Journal of Stored Products Research 39, 305-312

Pinotti L, Ottoboni M, Caprarulo V, Giromini C, Gottardo D., Cheli F., Fearn T., Baldi A. 2016. Microscopy in combination with image analysis for characterization of fishmeal material in aquafeed. Journal of Animal Feed Science and Technology, $215,156-164$.

Pinotti L, Fearn T, Gulalp S, Campagnoli A, Ottoboni M, Baldi A, Cheli F, Savoini G, Dell'Orto V, 2013. Computer image analysis: an additional tool for the identification of processed poultry and mammal protein containing bones. Food Addit, Contam. A 30, 1745-1751.

Quinn FA, Burkholder W, Kitto GB. 1992. Immunological technique for measuring insect contamination of grain. Journal of Economic Entomology 85, 1463-1470.

Rumpold BA, Schlüter OK. 2013. Potential and challenges of insects as an innovative source for food and feed production. Innovative Food Science and Emerging Technologies 17, 1-11.

Sánchez-Muros MJ, Barroso FG, Manzano-Agugliaro F. 2014. Insect meal as renewable source of food for animal feeding: a review. Journal of Cleaner Production 65, 16-27.

Schatzki TF, Wilson EK, Kitto GB, Behrens P, Heller I. 1993. Determination of hidden Sitophilus granarius (Coleoptera: Curculionidae) in wheat by myosin ELISA. Journal of Economic Entomology 86, 1584-1589. 
Tena N, Fernández Pierna JA, Boix A, Baeten V, von Holst C. 2014. Differentiation of meat and bone meal from fishmeal by near-infrared spectroscopy: Extension of scope to defatted samples. Food Control, 43, 155-162.

Thomas PA, Kaliamurthy J, Jesudasan CAN, Geraldine P. 2008. Use of chlorazol black E mounts of corneal scrapes for diagnosis of filamentous fungal keratitis. American Journal of Ophthalmology, 145 (6), 971-976. http://doi.org/10.1016/j.ajo.2008.01.020.

Van Huis A. 2013. Potential of insects as food and feed in assuring food security. Annu Rev Entomol 58, 563-583.

van Raamsdonk LWD, Veys P, Vancutsem J, Pridotkas G, Jørgensen JS. 2012. Classical microscopy. Improvements of the qualitative protocol. In: J.S. Jørgensen and V. Baeten (eds.) Detection, identification and quantification of processed animal proteins in feedingstuffs, Namur, Belgique. Les Éditions namuroises, Namur, Belgium, 47-57.

Veldkamp T, Bosch G. 2015. Insects: a protein-rich feed ingredient in pig and poultry diets. Animal Frontiers 5(2), 45-50.

Veys P, Berben G, Dardenne P, Baeten V. 2012. Detection and identification of animal by-products in animal feed for the control of transmissible spongiform encephalopathies. Animal Feed Contamination: Effects on Livestock and Food Safety, pp. 94-113. Woodhead Publishing

Watkins BE, Adair J, Oldfield JE. 1982. Evaluation of shrimp and king crab processing byproducts as feed supplements for mink. J Anim Sci, 55, 578-589. 
Table 1. Summary results: effectiveness of Alizarin Red and chlorazol black staining in terrestrial and marine arthropod materials tested. (-, absence of staining; +, variable staining intensity: ++ , strong staining.

\section{Alizarin Red Chlorazol black}

Hermetia illucens

Bombyx mori

Tenebrio molitor

$++$

Krill meal

(1)


Figure 1. Insect material observed under bright field conditions: A) Hermetia illucens fragment. AR stain. Embedding agent: Norland optical adhesive 65; B) Hermetia illucens fragment. CB stain. Embedding agent: Norland optical adhesive 65; C) Hermetia illucens fragment. Embedding agent: Norland optical adhesive 65. Arrow head indicates cell-like structures; D) Bombyx mori fragment. AR stain. Embedding agent: Glycerol. Arrow head indicates cell-like structures; E) Bombyx mori fragment. CB stain. Embedding agent: Glycerol; F) Bombyx mori fragment. Embedding agent: Glycerol; G) Tenebrio molitor fragment. AR stain. Embedding agent: Glycerol. H) Tenebrio molitor fragment. CB stain. Embedding agent: Glycerol; I) Tenebrio molitor fragment. Embedding agent: Glycerol.
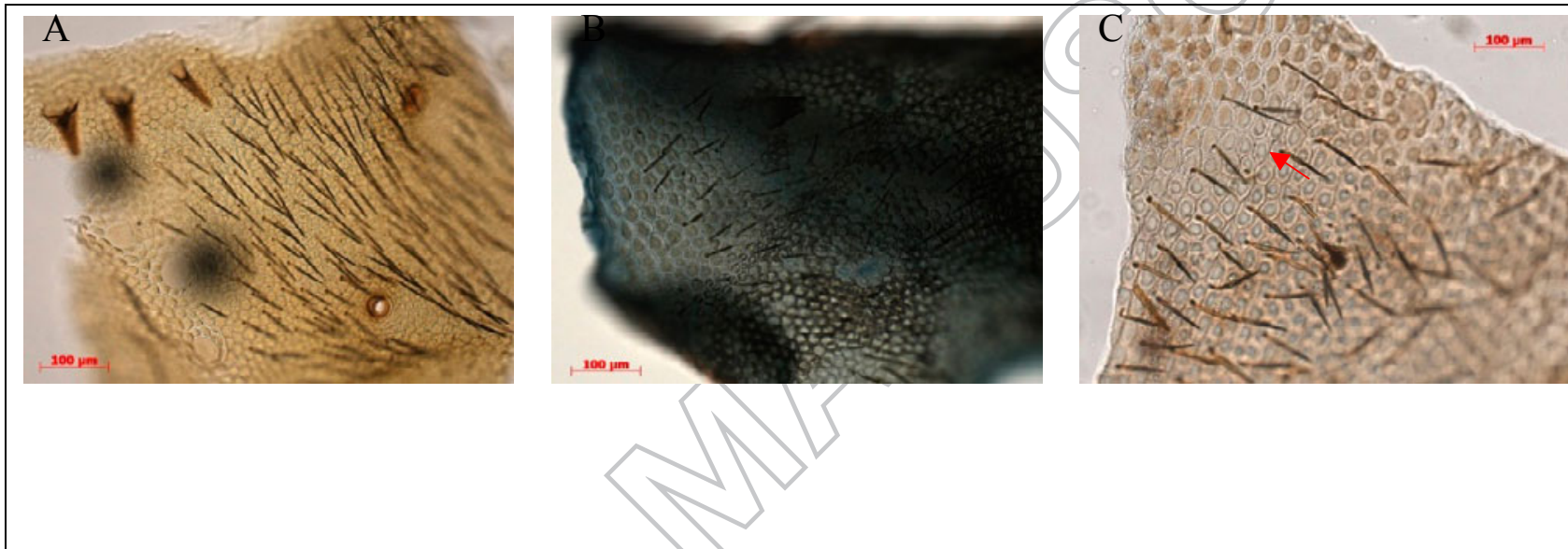

$\mathrm{D}$
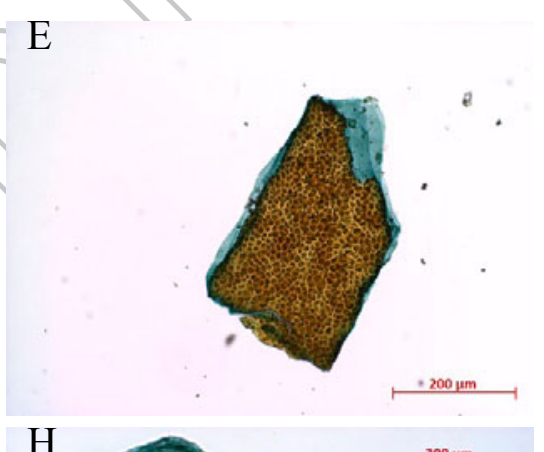

$\mathrm{F}$
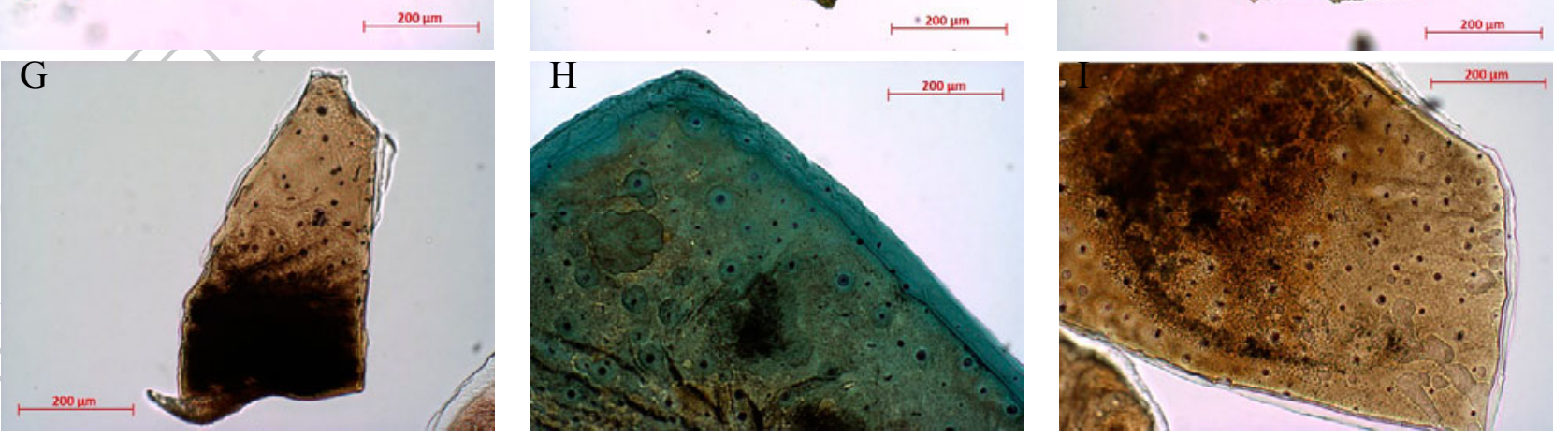
Figure 2. Marine arthropods materials observed under bright field conditions. A)

Shrimp meal fragment. AR stain. Embedding agent: Norland optical adhesive 65; B)

Shrimp meal fragment, CB stain. Embedding agent: Norland optical adhesive 65; C)

Shrimp meal fragment. Embedding agent: Norland optical adhesive 65; D) Krill meal fragment. AR stain. Embedding agent: Glycerol; E) Krill meal fragment. CB stain.

Embedding agent: Glycerol; F) Krill meal fragment. Embedding agent: Glycerol.

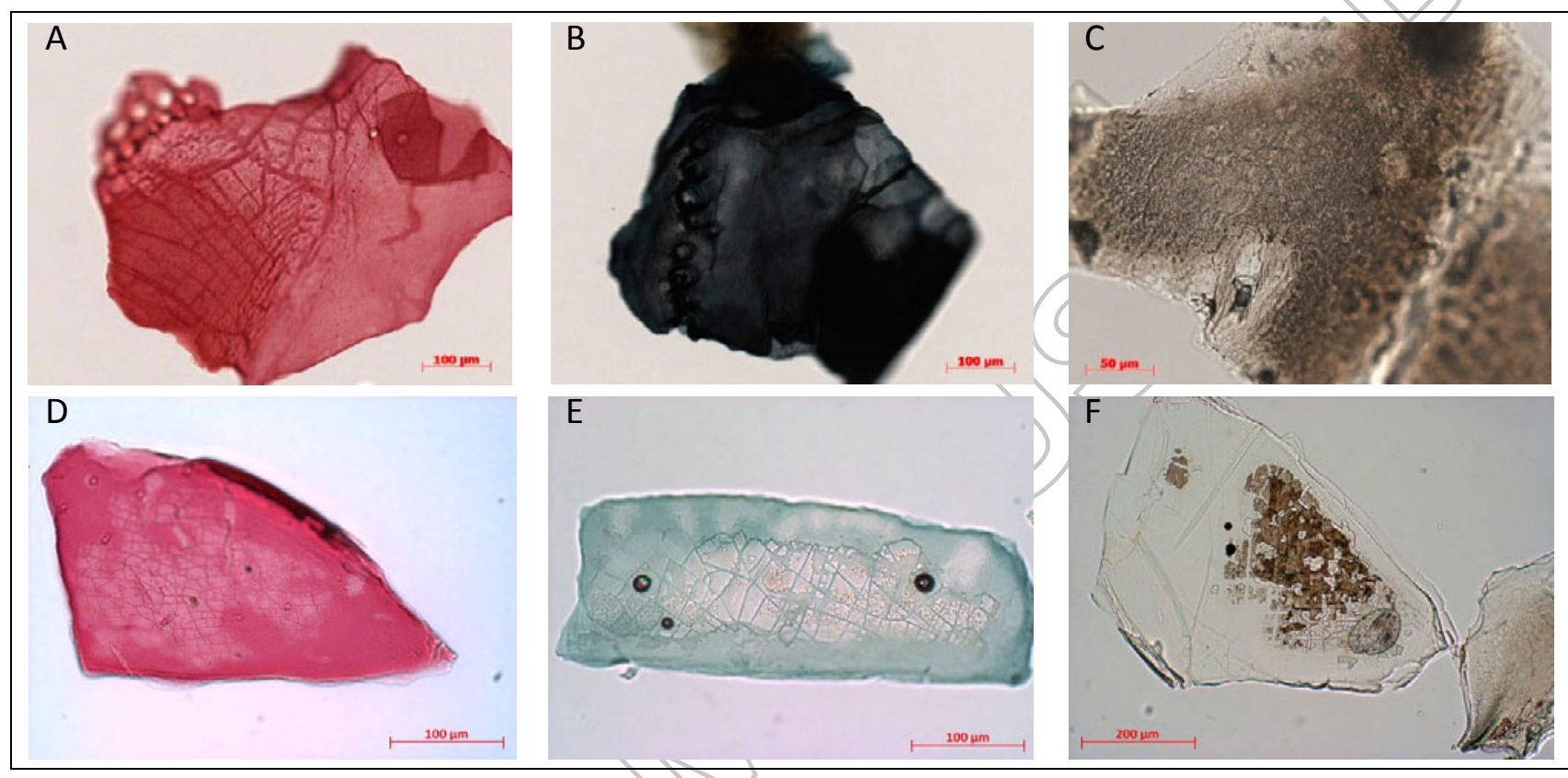

